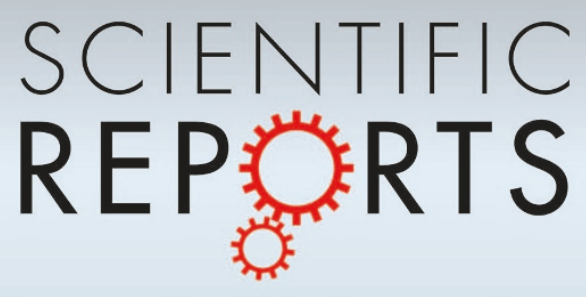

OPEN

SUBJECT AREAS:

COMPUTATIONAL

BIOLOGY AND

BIOINFORMATICS

DISEASES

BIOLOGICAL MODELS

COMPUTATIONAL MODELS

Received

23 May 2012

Accepted

2 November 2012

Published

16 November 2012

Correspondence and requests for materials should be addressed to K.G. (kagr@imm.dtu.

$\mathrm{dk})$

\section{Simulating spread of Bluetongue Virus by flying vectors between hosts on pasture}

\author{
Kaare Græsbøll1,2, René Bødker', Claes Enøe' \& Lasse E. Christiansen² \\ ${ }^{1}$ National Veterinary Institute, Technical University of Denmark, ${ }^{2}$ Department of Informatics and Mathematical Modelling, Technical \\ University of Denmark.
}

Bluetongue is a disease of ruminants which reached Denmark in 2007. We present a process-based stochastic simulation model of vector-borne diseases, where host animals are not confined to a central geographic farm coordinate, but can be distributed onto pasture areas. Furthermore vectors fly freely and display search behavior to locate areas with hosts. We also include wind spread of vectors, host movements, and vector seasonality. Results show that temperature and seasonality of vectors determines the period in which an incursion of Bluetongue may lead to epidemic spread in Denmark. Within this period of risk the number of infected hosts is affected by temperature, vector abundance, vector behavior, vectors' ability to locate hosts, and use of pasture. These results indicate that restricted grazing during outbreaks can reduce the number of infected hosts and the size of the affected area. The model can be implemented on other vector-borne diseases of grazing animals.
ulicoides-borne diseases represent a growing expense for the European dairy and meat industry ${ }^{1-3}$. The outbreak of Bluetongue in the temperate parts of northern Europe in 2006-2008 is one of the costliest examples hereof. Recently Culicoides species from temperate parts of Europe have been shown to carry the Schmallenberg Virus ${ }^{4}$, which is closely related to Akabane ${ }^{5}$; similarly to BTV, these two diseases have negative effects on ruminant production animals. Given the increased numbers of diseases carried by Culicoides it becomes important to develop strong decision-making tools to help assist in evaluating best practises against epidemic spread of vector-borne diseases. Such tools should be based on models that are able to predict the spread of disease with good precision.

Bluetongue virus (BTV) first occurred in Denmark in 2007. The arrival of the virus gave rise to only a few cases of BTV infection in that and the following year, however, a costly vaccination campaign was launched to prevent a wide-scale epidemic. Due to the relative shortness of the warm period in Denmark and the other Nordic countries, it has been hypothesized that the temporal window for BTV transmission may be so short in these countries that a large-scale epidemic is unlikely, and hence vaccination or other preventive measures may not be necessary.

The aim of the model presented in this paper is to describe the spread of vector-borne diseases with a high degree of spatial precision. The model should also be so fast that it can be used to examine several different scenarios should such a vector-borne disease enter the area of interest, and simulate this within a reasonable amount of (CPU) time.

Following the 2006 outbreak of BTV in northern Europe, models with increasing complexity have been created to model Bluetongue ${ }^{6-11}$. We elaborate on existing frameworks by introducing more realistic spatial parameters. Firstly, we assign host animals to pasture areas, and secondly, we simulate vector movements. By modeling the actual movements of vectors between pasture areas with and without hosts, we observe that spatial spread becomes more sensitive to parameters affecting vectors.

In the following we present more than 1,000,000 simulated years of Bluetongue in Denmark; the total data set represents less than 20 hours single CPU time.

\section{Results}

A major difference between our model and previous models is that in our model virus is transported between herds on pasture by vectors as opposed to between farm locations only. Therefore the area in which vectors can locate hosts with certainty becomes a large driving factor of epidemics. The simulations show that, when increasing the grid cell size, which represents the area in which vectors locate host animals, the number of affected cattle increases, as seen on rows in figure 1. In the literature there are no direct observations of how large the area 

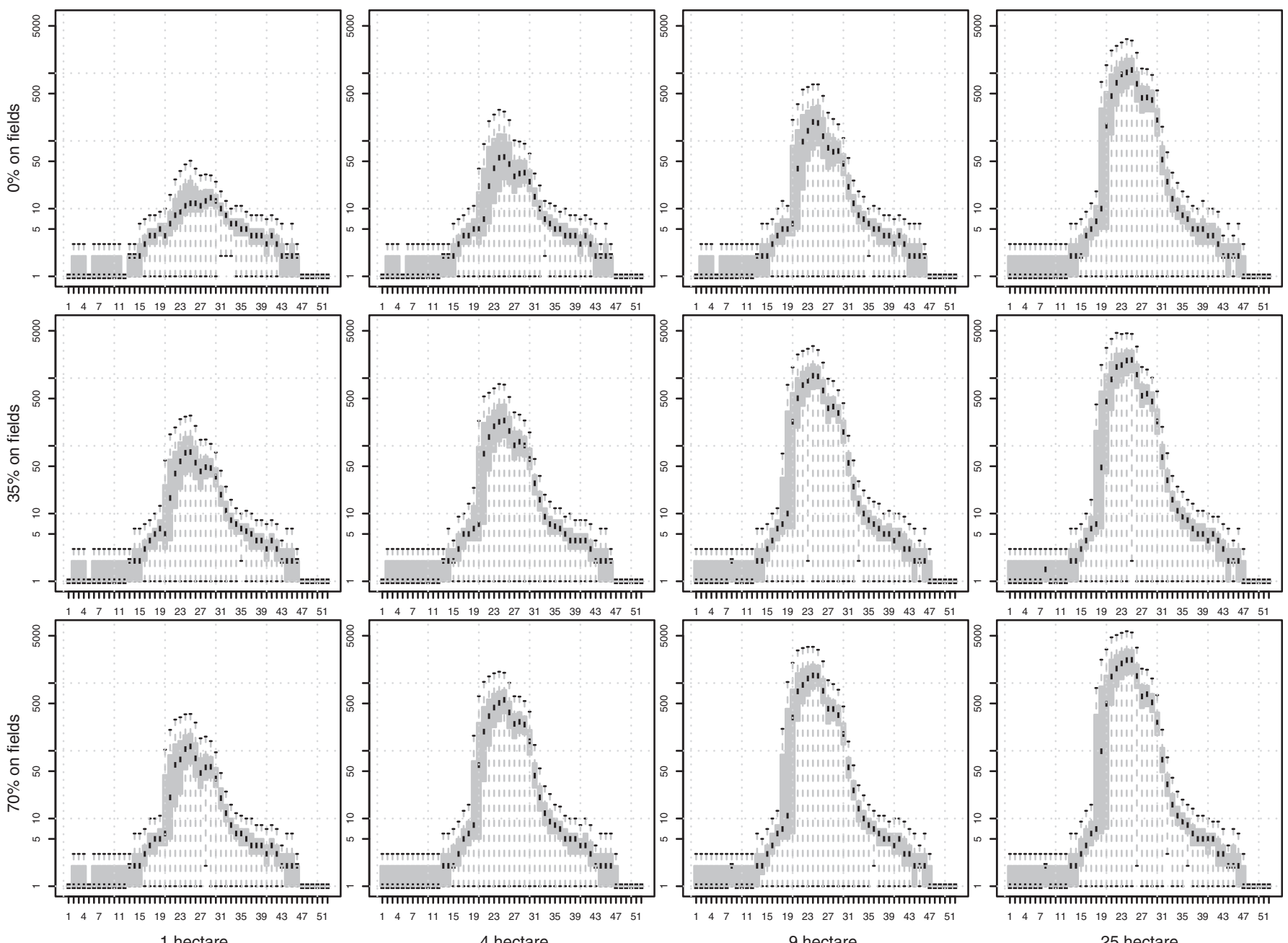

Figure 1 Number of affected cattle as a function of week of introduction of disease. Rows have cattle distributed from $0 \%$ of cattle grazing on fields (all within farm buildings) to $70 \%$ on fields. Columns have the area in which vectors are able to locate hosts ranging from 1 to 25 hectare. One is added to all data to use log-scale on the y-axis.

in which vectors will certainly locate hosts is. Based on mosquito data, Sedda et al ${ }^{10}$ estimated that midges can detect hosts that are up to 300 meters away. Another way of increasing the probability with which vectors locate hosts is to increase the proportion of farms with host animals on fields. This increases the overall area with hosts and therefore also the probability for vectors to locate hosts. This can be observed in the increased number of affected cattle when there is increased use of pasture (columns in figure 1). However, when the capability of vectors to locate hosts (the grid cell size) exceeds the average distance between neighboring farms, there will be no added effect of putting host to pasture because vectors can locate from farm to farm as easily as from pasture to pasture.

The number of affected animals generally correlates positively with the spread of the disease (compare figures 1 and 2). Notice that the colored pixels in figures 2 and 4 are aggregated data 5 by $5 \mathrm{~km}$ and does not represent the grid cell size chosen in the simulation. The beginning of the epidemic outbreak window coincides with the beginning of the vector active period in week 20 . Arrival of infectious vectors later than week 30 results in few disease incidences because the temperature is too low for the virus to complete its extrinsic incubation period in the vector. The combined effect of the short vector period and cold winters in Denmark results in a window of three months in which at least $50 \%$ of simulations show more than 500 cattle are affected with Bluetongue, given that at least $35 \%$ cattle are on pasture and vectors able to locate cattle on at least 9 hectares (figure 1). The total number of affected hosts is very sensitive to additional parameters which we will describe in the next paragraph.

Sensitivity of the model. We tested the sensitivity of several parameters. In figures 3 and 4 (a,b) the number of infected host animals and the spread of disease is seen to be very dependent on the temperature and the number of midges per host. Higher vector abundance introduces more susceptible bites to infectious hosts, and higher temperature increases the probability for the virus to complete the EIP in the vector. Therefore, both higher vector abundance and higher temperature result in an increase in the number of infectious vectors. The increase in the total number of affected hosts increases with almost one order of magnitude every time the abundance of vectors doubles or the temperature rises one degree.

For the high temperature and high vector abundance scenarios, we observe higher presence of disease in coastal regions, especially along the edge of the simulation box. Part of this increase is an effect of boundary conditions in the model. If a vector is sampled to move outside the simulation box, this move will be canceled, and therefore vectors close to the edges have lower probability of moving. The edges of the simulation box are the same as the edges of figures 2 and 4. Furthermore, as it is not fatal for vectors in the simulation to be in areas above water, we expect many of the vectors that blow out to sea will return, thereby also increasing the presence of disease in coastal areas. 


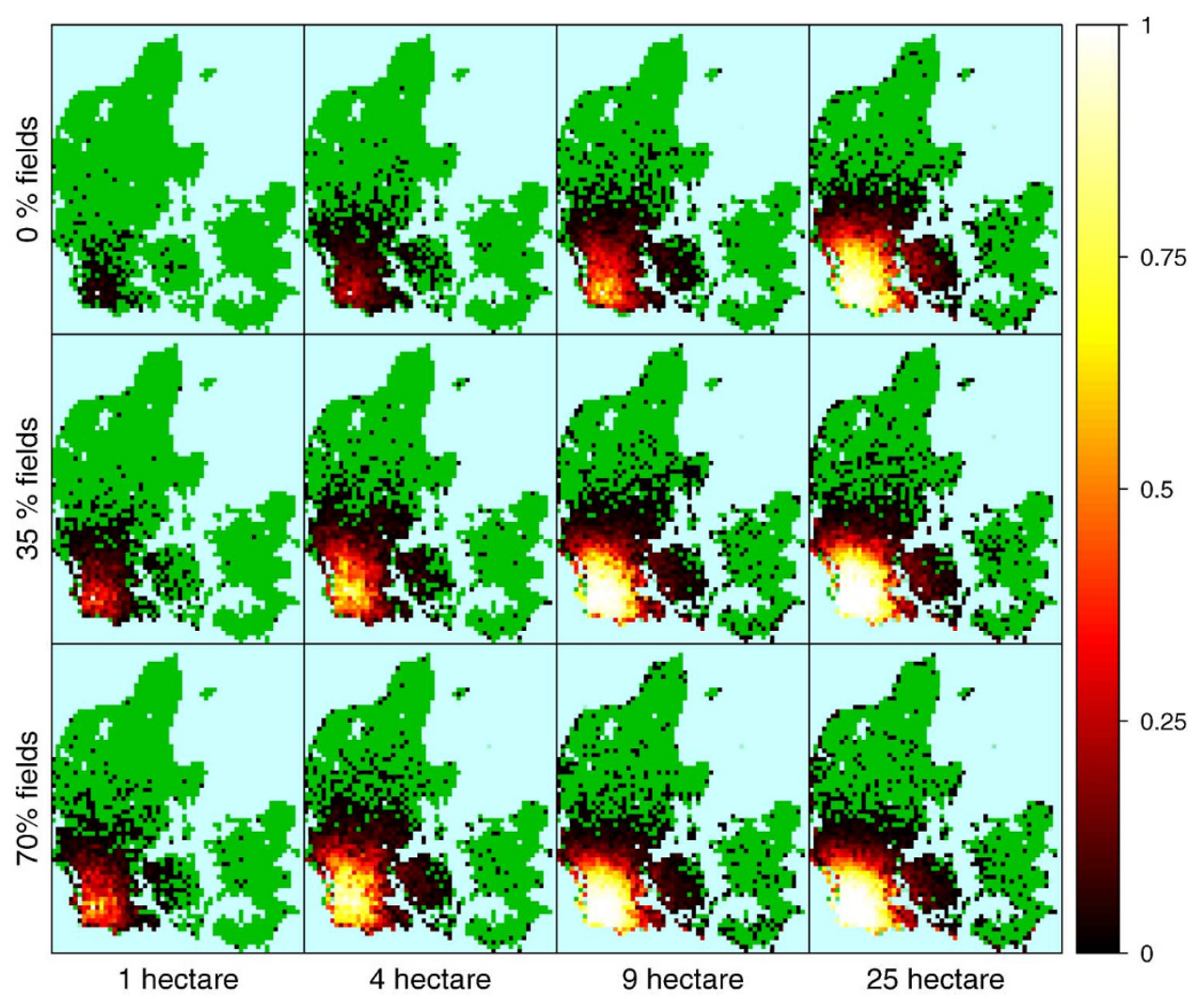

Figure 2 Influence of vector homing range and cattle distribution on spread of Bluetongue Virus. Fraction of simulations that show presence of disease within a 5 by $5 \mathrm{~km}$ pixel for introduction of infection at day 170 repeated 100 times. Rows have cattle distributed from $0 \%$ of cattle grazing on fields (all within farm buildings) to $70 \%$ on fields. Columns have the area in which vectors are able to locate hosts ranging from 1 to 25 hectare. The color green indicates pasture were no cattle were infected with Bluetongue. The color blue indicates area with no Danish pastures.

Figure $4(\mathbf{c}, \mathbf{d})$ indicates that longer flight ranges, whether active or wind-borne, increase the spatial spread of disease. However, this increased geographic spread is not reflected in the number of infected animals. From figure $3(\mathbf{c}, \mathbf{d})$ we observe that there are peak values in the number of affected hosts as a function of flying parameters. Beyond these peak values the simulations show fewer infected host animals. We therefore conclude that high dispersion of vectors may have a thinning effect on an epidemic outbreak (over-dispersion). This thinning effect, where the virus is spread over a greater area, but affects fewer hosts can be explained as follows: the daily probability of infecting a host is $\lambda=I_{V} a b / H$ (from table 1). For two herds with the same number of hosts, $H=H_{1}=H_{2}$, and susceptible hosts, $S_{H}=S_{H 1}$ $=S_{H_{2}}$, the probability that two infectious vectors present in one location, $I_{V 1}=2, I_{V 2}=0$, will not result in an infectious host is smaller than the probability that two infectious vectors in two different locations, $I_{V 1}=I_{V 2}=1$, will not result in an infected host, $\left(1-2 a b / H_{1}\right)^{S_{H 1}}(1-0)^{S_{H 2}}<\left(1-a b / H_{1}\right)^{S_{H 1}}\left(1-a b / H_{2}\right)^{S_{H 2}}$. The shorter the vectors fly the more probable it is that they land in the same place, and therefore the lower the risk of no infection of hosts. However, if they do not fly away from the place they acquired virus, with time the number of susceptible hosts $S_{H 1}$ will decrease because some of the hosts will become infected, and the inequality will no longer hold.

The sensitivity of the simulations to the probabilities of leaving areas with and without host animals $\left(\eta_{H}\right.$ and $\left.\eta_{! H}\right)$ are displayed in figures 3 and $4(\mathbf{e}, \mathbf{f})$, where we observe that the number of affected host animals is much more sensitive to whether vectors remain in a location with host animals, than to whether they leave areas without host animals. When a vector moves from a grid cell, the probability of it locating hosts is low (this probability varies with the distribution of hosts and is also geographically very different across Denmark because of differences in land use). As a result, the total number of affected cattle in the simulation is not very sensitive to changing the fraction of midges that fly from areas where there are no cattle, because most of these moves will result in landing in another cell without hosts. It is an a priori necessity for midges to fly from cells with hosts to achieve between-herd spread. Therefore figure $3 \mathrm{e}$ displays an increase in the number of affected hosts when increasing the fraction of vectors leaving cells with hosts. This upward trend peaks around $\eta_{H}=0.05 \mathrm{day}^{-1}$ because a large proportion of the infectious midges that fly away will never find new hosts, and therefore the number of affected hosts decreases if a large proportion of infectious vectors fly away instead of staying in the same grid cell and contributing to in-herd spread of disease.

With the exception of $\eta_{! H}$, we observe that the simulation model is very sensitive to all of the tested parameters (figures 1-4), either with regard to the total number of cases or with regard to the spatial spread of the disease.

We have not conducted sensitivity analysis on the duration of viremic period on cattle, since this parameter is well defined in literature. Furthermore, no sensitivity analysis was conducted on transmission probabilities $(b$ and $\beta)$. Given that these parameters influence the number of infectious bites in the exact same way as the number of vectors per host, we conclude that these parameters also influence outbreaks in the same way. Gubbins $(2008)^{7}$ performed uncertainty analysis on these in-herd parameters, which concluded that the uncertainties do not influence on the epidemic outbreak.

We tested the simulations with and without movement of infected host animals, but with the very low probability of movement, $p_{m}$, no substantial influence could be observed on the total number of affected host (results not shown). With host movement only a few long range spread events could be observed given that $86 \%$ of movements 

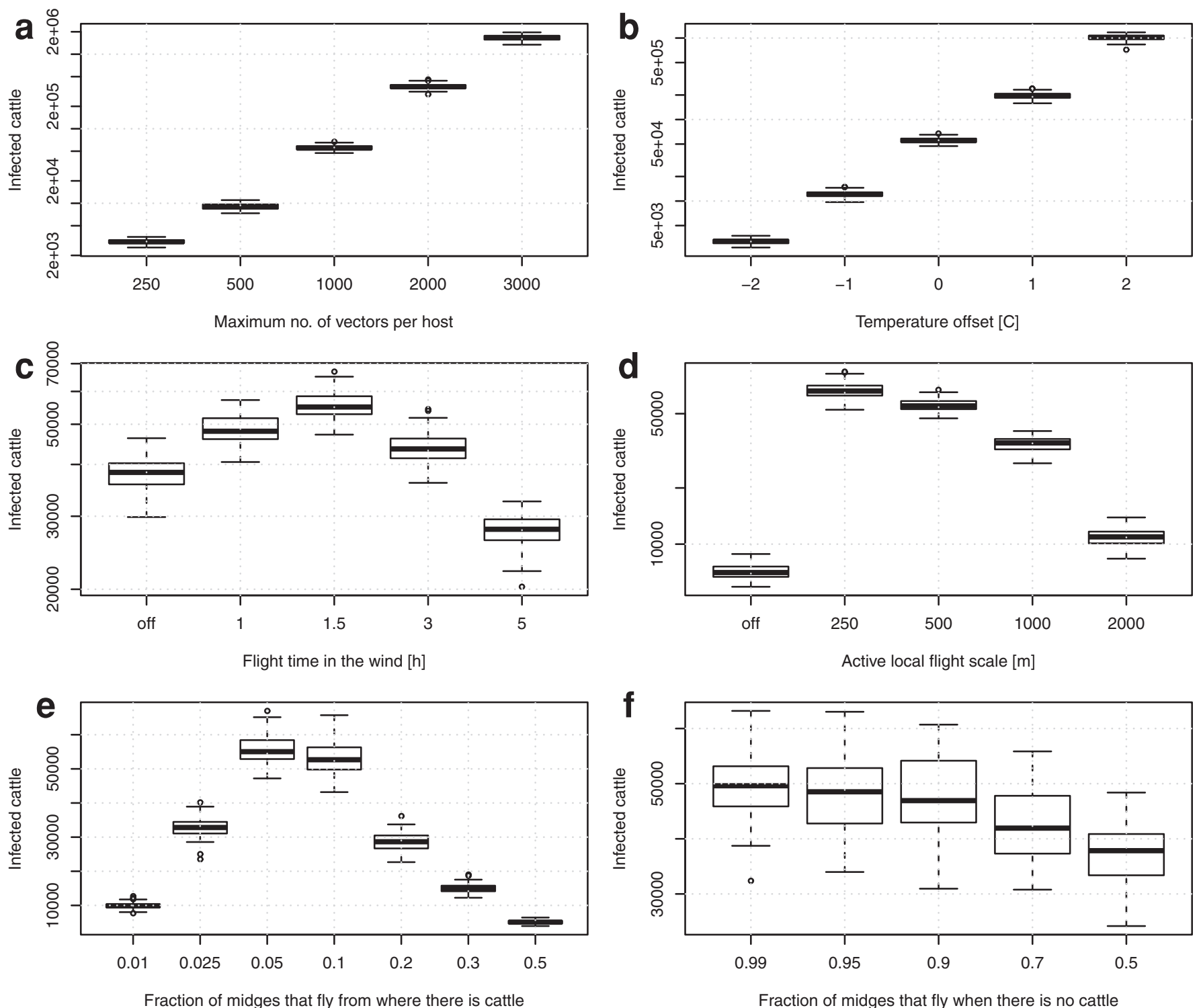

Figure 3 Sensitivity of simulations. The total number of infected cattle for infection day 1 through 365 repeated 100 times as a function of: (a) the maximum number of vectors to host, $m_{M}$; (b) the 2008 Danish temperatures with offset $\left[{ }^{\circ} \mathrm{C}\right] ;(\mathrm{c})$ the flight time in the wind [h], $t_{W} ;(\mathrm{d})$ the active local flight length $[\mathrm{m}], d_{L 50} ;(\mathrm{e})$ the fraction of vectors leaving area with hosts [1/day], $\eta_{H}$; and (f) the fraction of vectors leaving area with no hosts [1/day], $\eta_{! H}$. Grid cell size were 300 by $300 \mathrm{~m}$, with $35 \%$ of hosts on pasture.

are within $30 \mathrm{~km}$ and Danish cattle is in general not moved a lot compared to e.g. British sheep ${ }^{11}$.

It is generally observed that varying the parameters does not change the period in which introduction of virus would lead to an outbreak in Denmark. The exceptions to this observation are: that the end of the vector free period determines when onset of an epidemic is possible, and that the temperature determines when vectors can no longer become infectious. In this study we do not include the possible effects of rising temperatures on the vector abundance; warmer temperatures may lead to an earlier onset of vector season, with a wider window of opportunity for an epidemic.

\section{Discussion}

From this study we conclude that a large-scale epidemic is possible in Denmark given certain parameter values such as high temperature, high midge abundance, and ease with which vectors can locate host animals. But incursion of such an epidemic is limited by a time window which is dependent on vector period and temperature. The simulations show that incursions of BTV later than August cannot give rise to large epidemics. This is in agreement with the observed incursion of Bluetongue in Denmark in 2007, where one index case in October did not lead to any further spreading of the virus. Also an introduction in late August 2008 lead only to 15 known infected herds, with total 27 animals affected. However, the detected incursion in 2008 happened during the beginning of the Danish vaccination campaign against $\mathrm{BTV}^{12}$, which makes this incidence inconclusive evidence against the possibility of an epidemic outbreak.

Our simulations cannot predict the scale of an epidemic outbreak of Bluetongue in Denmark because the parameters used to describe vector behavior are not very well-defined. Therefore, we cannot conclude on the best prevention strategy for Denmark. The simulations do indicate that a warmer year than 2008 , or a warmer climate, will increase the size of epidemics. Furthermore, reducing the probability of vectors locating hosts reduces the total number of affected hosts in an outbreak. This can e.g. be achieved by moving host animals into stables should an outbreak occur. Future experiments will hopefully help us to better determine the flying parameters of midges, in order to make better predictions on epidemic sizes. 

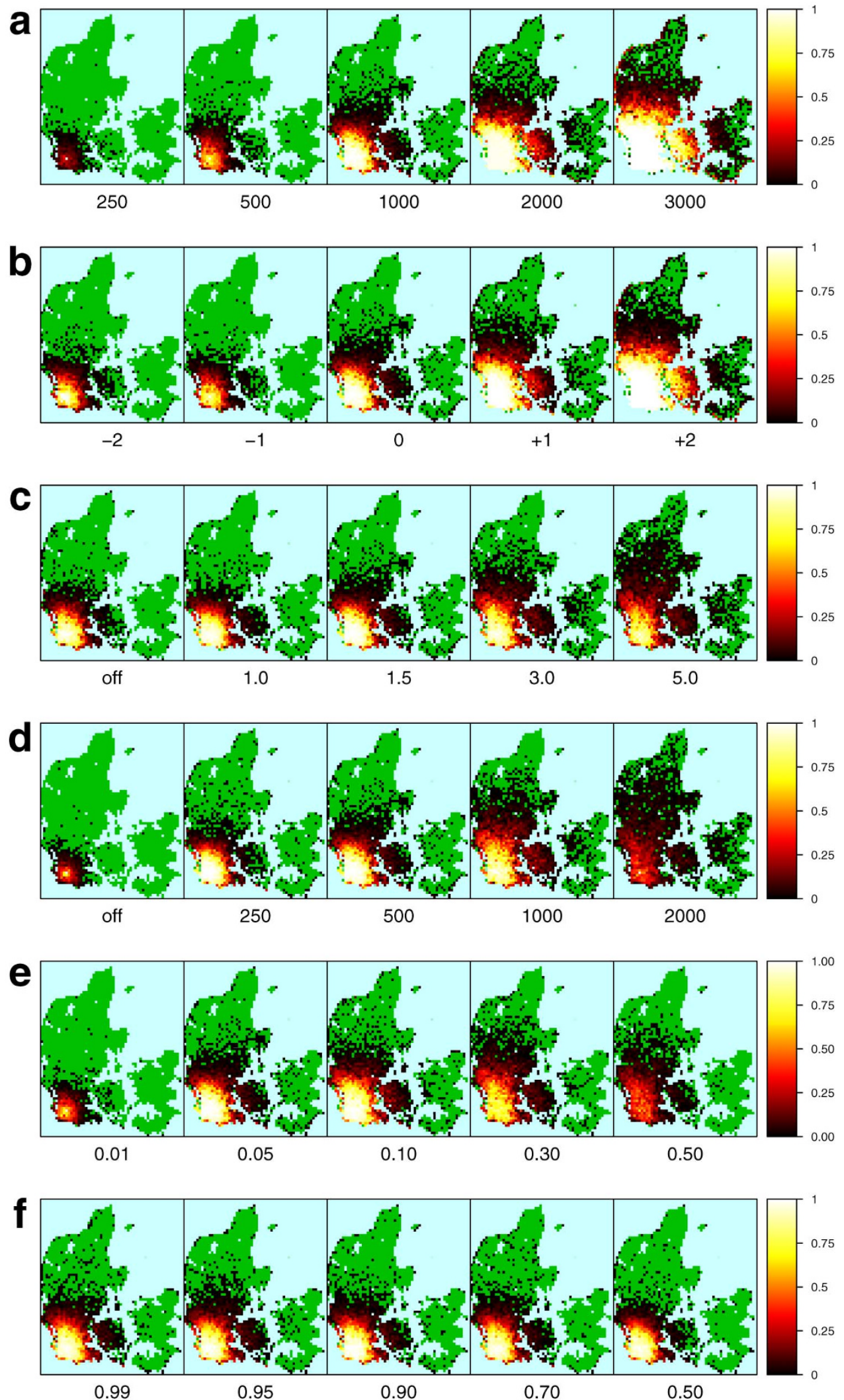

Figure $4 \mid$ Spread of Bluetongue virus. Fraction of 5 by $5 \mathrm{~km}$ pixels that show presence of virus for infection day 170 repeated 100 times as a function of: (a) the maximum number of vectors to host, $m_{M}$; (b) the 2008 Danish temperatures with offset $\left[{ }^{\circ} \mathrm{C}\right] ;(\mathrm{c})$ the flight time in the wind [h], $t_{W} ;(\mathrm{d})$ the active local flight length $[\mathrm{m}], d_{L 50}$; (e) the fraction of vectors leaving area with hosts [1/day], $\eta_{H}$; and (f) the fraction of vectors leaving area with no hosts [1/day], $\eta_{! H}$. Grid cell size were 300 by $300 \mathrm{~m}$, with $35 \%$ of hosts on pasture. The color green indicates pasture were no cattle were infected with Bluetongue. The color blue indicates areas with no Danish pastures. 
symbol value [range]

\begin{tabular}{|c|c|}
\hline $\bar{T}$ & data \\
\hline$H$ & data \\
\hline $\begin{array}{l}a \\
b\end{array}$ & $\begin{array}{c}0.0002 T(T-3.7)(41.9-T)^{0.37} \\
0.9\end{array}$ \\
\hline$\beta$ & 0.1 \\
\hline$m$ & $m_{M}\left|\sin \left(\frac{t-150}{40} \pi\right) \sin \left(\frac{t-150}{160} \pi\right)\right| *$ \\
\hline$m_{M}$ & 1000 \\
\hline $\begin{array}{l}1 / r \\
n\end{array}$ & 20.6 \\
\hline $1 / v$ & $v(T)=0.018(T-13.4)[1-100]$ \\
\hline $\begin{array}{l}k \\
\mu\end{array}$ & $0.5 / v[3-11]$ \\
\hline$\mu$ & $0.009 \exp (0.1607)[0.05-0.9]$ \\
\hline$\eta_{H}$ & 0.05 \\
\hline$\eta_{! H}$ & 0.95 \\
\hline$t_{W}$ & 1.5 \\
\hline$d_{L 50}$ & 0.5 \\
\hline 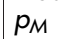 & 0.001 \\
\hline
\end{tabular}

description [unit]

references

temperature in DK by $25 \mathrm{~km}$ grid

herd size

biting rate $[1 /$ days $]$

probability of transmission from vector to host

probability of transmission from host to vector

Seasonality of vectors

22

[23]

$[7,24,25]$

[21]

maximum no. of vectors per host

mean duration of host viraemia [days]

stages in viremic period for hosts

mean extrinsic incubation period (EIP) [days]

stages to describe EIP in vector

vector mortality rate

probability of vector leaving area with hosts

probability of vector leaving area with no hosts

time vectors stay in the wind [hours]

median length vectors will fly at random $[\mathrm{km}]$

daily probability of hosts movement [1/herd]
[26]

$[26]$

Expressions dependent on temperature are imposed [range] limits shown in the table.

"For $150 \leq t \leq 3100$ for $t$ otherwise.

Despite these reservations this new simulation model has proven to be highly sensitive to all parameters, which is desirable if input of sufficient quality can be provided. Given that many parameters, such as the flying parameters, and both host and vector distributions, are often poorly described, we can only conclude on results that are robust across parameter values. But with the increased focus on biting midges as vectors more parameters should soon be available. Given that the model relies on data reported from the EU, this model can be extended to all of Europe as it can incorporate the differences in climate and herd distribution across the EU.

The novel approach taken here of simulating midges as agents of spread of the disease has lead to the spread of virus in this model being highly sensitive to parameters affecting midges. We observe that adjusting parameter values so that they produce more infectious midges causes more infected hosts and a wider spatial spread of disease. Furthermore this type of process based modeling allows to include actual wind data, which can better represent the spatial spread of epidemics given that many areas have predominant wind directions. However to improve the predictive value of our and similar models requires much more information about the flight behavior of vectors.

\section{Methods}

Modeling vector-borne non-contagious diseases has two primary tasks: To describe the in-herd spread and the between-herd spread. In this paper we define a herd as any group of suitable hosts for the disease which is spatially discernible from other groups of hosts. This definition results in farm holdings often being split into several herds when put to pasture.

Distribution of herds. Denmark was divided into grid cells, where the cell side length is an input parameter ranging from 100 to 500 meters. In each grid cell, a number of hosts and vectors can be assigned. The vectors can localize all the hosts within the same grid as itself, thus the grid size in the simulation is essentially a measure of the vectors' ability to locate host animals. The hosts within one grid cell belongs to one herd.

To distribute hosts on pasture in Denmark, we combined information from the Danish Central Husbandry Register (CHR) with data from the EU arable land subsidies program linking cattle farmers with potential pasture areas. From this data, $70 \%$ of cattle owners were identified as registered landowners of grass-covered areas. This information made it possible to model different 'host on pasture' scenarios ranging from 'all hosts staying inside farm buildings' up to ' $70 \%$ of cattle farmers distributing hosts on pasture'. As we do not know exactly which farmers do and do not put their animals out to grass, we randomly sampled which farmers had put animals to pasture for every simulation. Cattle selected for pasture were distributed into the grid cells covering pasture owned by their respective farmers. All animals not put out to pasture were placed in the grid cell containing the coordinate of the owner's farm. The data set includes 21,877 farms with 1.6 million cattle, of which 16,364 owned 89,756 fields covering a total of 2.0 million hectare. We have not included sheep or goats in this simulation given that cattle outnumbers these by more than ten to one.

The simulations cover all of Denmark with the exception of the island of Bornholm, which was excluded due to its epidemiologically isolated location in the Baltic Sea.

Between-herd spread. There are three principal ways in which the disease can move between grids in the simulation: transportation of host animals, active flight by vectors, and passive (wind-borne) flight of vectors.

Transportation of animals was modeled using a distance kernel. Which states the daily probability of a herd having hosts moved, $p_{M}$, and specifies the distance $[\mathrm{km}]$ hosts were moved, $d_{M}$, should such a move occur. The parameters were extracted from the movement register in Denmark, which tracks all movements of cattle.

$$
d_{M}(x)=12 \log ^{2}\left(\frac{1.01-x}{1.2}\right)
$$

Where $x$ is drawn from a uniform distribution on ]0; 1]. Data on movements were from the period October 2006 to September 2007, and therefore this data is not affected by the movement restrictions implemented after the discovery of Bluetongue Virus in Denmark. Movement restrictions followed the EU guidelines with regard to BTV (ec.europa.eu).

Within each grid cell it is assumed that the vectors can locate the host animals with $100 \%$ certainty. Beyond the size scale of the grid, vectors are assumed to fly a random walk pattern to locate hosts. A random walk can be described by a Gaussian spread kernel, and the flight distance of a vector, $d_{L}$, can thus be drawn using the normal distribution in equation (2) as the frequency distribution, equation (3).

$$
\begin{gathered}
f_{G}(x ; \mu, \sigma)=\frac{1}{\sigma \sqrt{2 \pi}} \exp \left(-\frac{(x-\mu)^{2}}{2 \sigma^{2}}\right) \\
f\left(d_{L}\right)=2 f_{G}\left(d_{L} ; 0, \frac{d_{L 50}}{P_{50}}\right)
\end{gathered}
$$

Where the constant $P_{50}=0.675$ scales so that $d_{L 50}$ becomes the radial distance $50 \%$ of the vectors will fly within. The factor of two normalizes the distribution given that distances cannot be negative.

Similarly for wind-borne flight, $d_{W}$, the wind carries vectors, and the distance carried is drawn again using the Normal distribution as frequency distribution.

$$
f\left(d_{W}\right)=2 f_{G}\left(d_{L} ; 0, \frac{d_{W 50}}{P_{50}}\right), \quad d_{W 50}=t_{W} w_{S}
$$

Where $d_{W 50}$ is the distance $50 \%$ of the vectors are carried within by the wind. This is a product of the wind speed, $w_{S}$, and the time vectors spend in the wind, $t_{W}$.

The direction of the active flight is drawn from a uniform distribution. While for wind dispersal the direction, $\theta_{W}$, is distributed as a Gaussian centered on the wind direction, $w_{\theta}$

$$
f\left(\theta_{W}\right)=w_{\theta}+f_{G}\left(\theta_{W} ; 0, \frac{\theta_{c}}{P_{90}}\right)
$$

Where $\theta_{c}=\pi / 4$ and $P_{90}=1.645$ are given so that $90 \%$ of the vectors are within a cone spanning $45^{\circ}$ around the wind direction. The direction of the wind used in this paper 


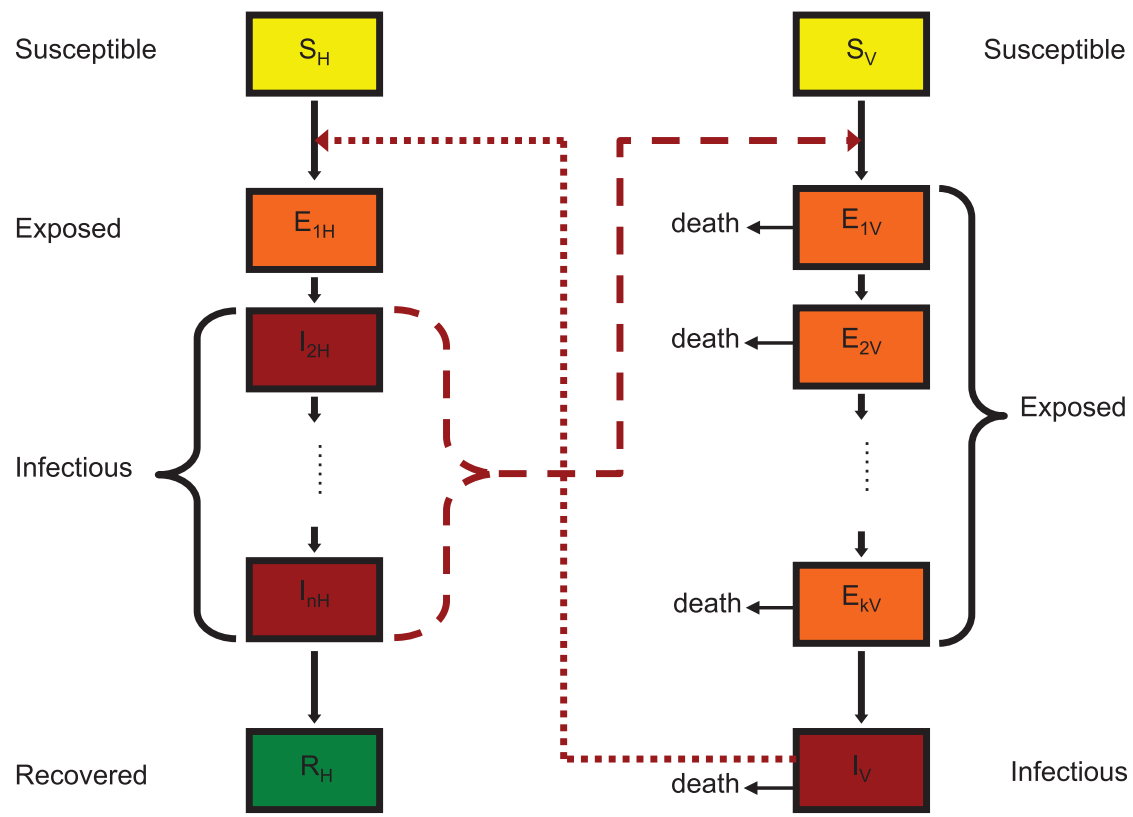

Figure $5 \mid$ Dynamic of disease. The viraemia of the hosts is described by an extended SEIR model, and the vectors are described by an extended SEI model. All movements between the stages in the model are governed by the probabilities listed in tables $1-2$.

was randomly sampled once daily, and the wind speed was sampled uniformly from $0-5 \mathrm{~m} / \mathrm{s}$. Future versions of the program will accept input of actual wind data. We do not expect actual wind data to influence much on the size of outbreaks. However, real wind data will probably influence the spatial extent and shape of the outbreak.

Both active and passive vector movement are applied on each individual vector in each grid cell.

Simulated vectors in this model are "free" to disperse in a series of jumps before transmitting the disease. As opposed to traditional kernel models where transmission events are determined by the kernel. Free dispersal in combination with splitting vector movements into two separate Gaussians makes it possible for transmission events to occur as observed in real epidemics both short, long and fat tails as dependent by regional farming practices or policies ${ }^{13}$.

We introduce two parameters to describe the behavior of vectors in grid cells with and without host animals. The daily probability of vectors leaving cells with no host $\eta_{: H}=0.95 \mathrm{day}^{-1}$, is set at a high value to reflect that the evolutionary pressure to locate blood meals is very high. When a stable source of blood meals is discovered, there is little gain in flying away, so the daily probability of leaving an area with host animals is set at a low value, $\eta_{H}=0.05 \mathrm{day}^{-1}$. When we assume that midges prefer to stay in grid cells with hosts, we implicitly assume that there are suitable breeding grounds within the grid cell or that vectors have a homing behavior, which enables them to migrate between the same herd and their breeding sites. The validity of these assumptions depends on the behavior of the vectors, and Culicoides are known to display different breeding patterns depending on species ${ }^{14,15}$. Other models without active movement of vectors generally have an implicit $\eta_{H}=0$, because virus is not removed from herds in case of transmission events.

In-herd spread. Within each herd, the dynamics work in almost the same way as described in previous articles relating to Bluetongue modeling ${ }^{7,9}$. Hosts are described using an extended Susceptible Exposed Infectious Recovered (SEIR) type framework, while vectors are described using an extended Susceptible Exposed Infectious (SEI) framework (figure 5). The extended framework ensures that the model emulates virus incubation time more realistically compared with the non-extended approach. The SEI/SEIR framework uses rates which assign a probability of movement of an

\begin{tabular}{|lll|}
\hline \multicolumn{3}{l}{ Table 2 | Transition probabilities between states in figure 5 } \\
probability of & expression & transition \\
\hline infecting host & $a b I_{V} / H$ & $S_{H} \rightarrow E_{1 H}$ \\
host becoming infectious & $n r$ & $E_{1 H} \rightarrow I_{2 H}$ \\
host moving through stages & $n r$ & $I_{(i-1) H} \rightarrow I_{i H} i=3,4, \ldots, n$ \\
host recovering & $n r$ & $I_{n H} \rightarrow R_{H}$ \\
infecting vector & $a \beta \sum_{2}^{n} I_{i H} / H$ & $S_{V} \rightarrow E_{1 V}$ \\
vector moving through stages & $k v$ & $E_{(i-1) V} \rightarrow E_{i V} i=2,3, \ldots, k$ \\
vector becoming infectious & $k v$ & $E_{n V} \rightarrow I_{V}$ \\
\hline
\end{tabular}

individual vector/host from one state to the next within one time step. In the original unextended SEIR model $(k=1)$ most midges become infectious on the time step immediately after obtaining the virus, which in the case of Bluetongue (and many other vector borne diseases) is impossible in nature, especially in areas with low temperature resulting in a long extrinsic incubation period (EIP). Therefore the exposed states of vectors are modeled using extra stages to better emulate the incubation period. These extra stages bring the frequency distribution of vectors completing the incubation period from an exponential distribution to a Erlang (Gamma) distribution ${ }^{16}$. When adding a risk of dying to the system, the frequency of vectors completing the incubation period becomes a phase-type distribution ${ }^{17}$. We also uses an extended approach on the infectious period of the host to better describe the viremic period.

Figure 5 depicts the stages in the extended SEI/SEIR model. The arrows indicate an event described by parameters and transition probabilities in table 1 and 2 .

Bluetongue is a non-contagious disease and therefore transmission of virus is only possible through the bite of the vectors.

Vector abundance. The spread of vector borne diseases is highly sensitive to the abundance of vectors, but the vector abundance is often not well determined. For the Bluetongue vector there are numerous papers on catches of midges; where in the field one may catch thousands of midges one night and close to zero on the next. Therefore modeling abundance is often difficult and most be done over longer timescales to account for the large variability in daily trap catches ${ }^{18-20}$.

The seasonal midge abundance, $m$, in Denmark across the year (table 1 ) is adopted from Nielsen (1996) $)^{21}$. The abundance of vectors is represented by the absolute of a sinusoidal curve to emulate four generations from day 150 to 310 in the year. The estimate of $m_{M}$ represents the maximum abundance of vectors which is multiplied onto the seasonality to give the daily maximum abundance. We sample the number of susceptible vectors uniformly from zero to daily maximum each day at each location to account for the day to day variation in midge catch that is observed in the original studies.

Simulations. All simulations were run for one year from 1 January to 31 December using temperature data from 39 selected meteorological stations recorded in 2008. Data from the 39 weather stations was interpolated to a grid of 25 by $25 \mathrm{~km}$ (71 grid points) in connection with another project (www.nordrisk.dk), and each herd used the temperature from nearest grid point. In the simulation disease is introduced by 10 infectious midges that arrive in the southern part of Jutland (the large landmass in the western part of Denmark). The date of arrival is repeated 100 times across the whole year for each of the 12 scenarios for distribution of host and search area of vectors, in total $365 \cdot 100 \cdot 12=438,000$ years simulated for the data in figure 1 . The point of arrival can be seen as a white square in the plots in figure 2. However, the exact spot of arrival can vary slightly between scenarios because we required the spot of arrival to have host animals, and presence of host animals depends on the distribution of hosts on pasture.

The introduction point in southern Jutland was chosen given that both Bluetongue (2009) and Schmallenberg virus (2012) have been previously introduced in this area 
(See the OIE WAHID database). Furthermore this area have the highest density of cattle in Denmark, and is therefore considered of highest risk. The climate in Denmark is very homogenous and introducing virus in other parts of Denmark gives similar results (not shown), although scaled with the regional host density.

Sensitivity analysis was done on the scenario with grid cell size 300 by $300 \mathrm{~m}$ and $35 \%$ of hosts on pasture. The tested outcome was the total number of affected hosts for a period of $365 \cdot 100=36,500$ years of simulated outbreaks per parameter value.

1. Dufour, B., Moutou, F., Hattenberger, A. M. \& Rodhain, F. Global change: impact, management, risk approach and health measures-the case of Europe. Rev. - Off. Int. Epizoot. 27, 529-550 (2008).

2. Wilson, A. J. \& Mellor, P. S. Bluetongue in Europe: past, present and future. Philos. Trans. R. Soc. Lond., B, Biol. Sci. 364, 2669-2681 (2009).

3. Gibbens, N. Schmallenberg virus: a novel viral disease in northern Europe. Vet. Rec. 170, 58 (2012).

4. Rasmussen, L. et al. Culicoides as vectors of schmallenberg virus [letter] Emerging Infectious Diseases (2012).

5. Hoffmann, B. et al. Novel orthobunyavirus in Cattle, Europe, 2011. Emerging Infect. Dis. 18(3), 469-472 (2012)

6. Guis, H. et al. Use of high spatial resolution satellite imagery to characterize landscapes at risk for bluetongue. Vet. Res. 38, 669-683 (2007).

7. Gubbins, S., Carpenter, S., Baylis, M., Wood, J. L. \& Mellor, P. S. Assessing the risk of bluetongue to uk livestock: uncertainty and sensitivity analyses of a temperature-dependent model for the basic reproduction number. Journal of The Royal Society Interface 5(20), 363-371 (2008).

8. Hartemink, N. et al. Mapping the basic reproduction number ( $\mathrm{r} 0$ ) for vectorborne diseases: A case study on bluetongue virus. Epidemics 1(3), 153-161 (2009).

9. Szmaragd, C. et al. A modeling framework to describe the transmission of bluetongue virus within and between farms in great britain. PLOS ONE 4(11), e7741 (2009)

10. Sedda, L. et al. A new algorithm quantifies the roles of wind and midge flight activity in the bluetongue epizootic in northwest Europe. Proc. Biol. Sci. 279(1737), 2354-2362 (2012).

11. Turner, J., Bowers, R. G. \& Baylis, M. Modelling bluetongue virus transmission between farms using animal and vector movements. Sci. Rep. 2 (2012).

12. Danish ministry of food, agriculture, and fisheries. www.foedevarestyrelsen.dk/ english/Animal/AnimalHealth/Bluetongue/Pages/Danish_bluetongue_outbreaks_ in 2008.aspx (2012).

13. de Koeijer, A. et al. Quantitative analysis of transmission parameters for bluetongue virus serotype 8 in western europe in 2006. Veterinary research 42(1), 1-9 (2011)

14. Kirkeby, C., Bødker, R., Stockmarr, A. \& Enøe, C. Association between land cover and culicoides (diptera: Ceratopogonidae) breeding sites on four danish cattlefarms. Entomologica Fennica 20(4), 228-232 (2009).

15. Ninio, C., Augot, D., Dufour, B. \& Depaquit, J. Emergence of culicoides obsoletus from indoor and outdoor breeding sites. Veterinary Parasitology 183(12), 125-129 (2011).

16. Carpenter, S. et al. Temperature dependence of the extrinsic incubation period of orbiviruses in culicoides biting midges. PLoS ONE 6(11), e27987 (2011).

17. Bladt, M. A review on phase-type distributions and their use in risk theory. Astin Bulletin 35(1), 145-161 (2005)

18. Nielsen, S., Nielsen, B., Axelsen, J. \& Fotel, F. Aktivitet af mitter på græsningsarealer ved egeløkke lung. Technical report, Miljøstyrelsen, (2003).

19. Sanders, C. J. et al. Influence of season and meteorological parameters on flight activity of culicoides biting midges. Journal of Applied Ecology 48(6), 1355-1364 (2011)
20. Ortega, M. D., Mellor, P. S., Rawlings, P. \& Pro, M. J. The seasonal and geographical distribution of Culicoides imicola, C. pulicaris group and C. obsoletus group biting midges in central and southern Spain. Arch. Virol. Suppl. 14, 85-91 (1998).

21. Nielsen, S., Nielsen, B., Axelsen, J. \& Fotel, F. Forurening af egeløkke lung og opformering af mitter. Technical report, Miljøstyrelsen, (1996).

22. Mullens, B. A., Gerry, A. C., Lysyk, T. J. \& Schmidtmann, E. T. Environmental effects on vector competence and virogenesis of bluetongue virus in Culicoides: interpreting laboratory data in a field context. Vet. Ital. 40, 160-166 (2004).

23. Baylis, M., O'Connell, L. \& Mellor, P. S. Rates of bluetongue virus transmission between Culicoides sonorensis and sheep. Med. Vet. Entomol. 22, 228-237 (2008)

24. Carpenter, S., Lunt, H. L., Arav, D., Venter, G. J. \& Mellor, P. S. Oral susceptibilit to bluetongue virus of Culicoides (Diptera: Ceratopogonidae) from the United Kingdom. J. Med. Entomol. 43, 73-78 (2006).

25. Carpenter, S. et al. Experimental infection studies of UK Culicoides species midges with bluetongue virus serotypes 8 and 9. Vet. Rec. 163, 589-592 (2008).

26. Melville, L. et al. Characteristics of naturally-occurring bluetongue viral infections of cattle. In Bluetongue Disease in Southeast Asia and the Pacific., St George, T. \& Peng, K., editors, Canberra: ACIAR proceedings series, 245-250 (1996).

27. Gerry, A. C. \& Mullens, B. A. Seasonal abundance and survivorship of culicoides sonorensis (diptera: Ceratopogonidae) at a southern california dairy, with reference to potential bluetongue virus transmission and persistence. Entomological Society of America 37, 675-688 (2000).

\section{Acknowledgments}

The authors greatly appreciate the Danish Knowledge Center for Agriculture, Dairy \& Cattle farming, for providing farm and movement data from the Danish cattle data base and for providing part of the funding. The Danish Meteorological Institute for contributing to the project with temperature data from the national climate stations. And the Faculty of Agricultural Sciences at Aarhus University for providing pasture data. Permission to merge these data sets was granted by the Danish Data Protection Agency on 1 July 2008. This study was partially funded by the EU grant FP7-261504 EDENext and is catalogued by the EDENext Steering Committee as EDENext046 (http://www.edenext.eu). The contents of this publication are the sole responsibility of the authors and do not necessarily reflect the views of the European Commission.

\section{Author contributions}

Conceived and designed the model: KG. Inputs on optimizing and structure of the model: LC. Inputs on biological behavior: RB. Wrote the paper: KG. Critically reviewed the paper: LC RB CE.

\section{Additional information}

Competing financial interests: The authors declare no competing financial interests.

License: This work is licensed under a Creative Common Attribution-NonCommercial-ShareALike 3.0 Unported License. To view a copy of this license, visit http://creativecommons.org/licenses/by-nc-sa/3.0/

How to cite this article: Græsbøll, K., Bødker, R., Enøe, C. \& Christiansen, L.E. Simulating spread of Bluetongue Virus by flying vectors between hosts on pasture. Sci. Rep. 2, 863; DOI:10.1038/srep00863 (2012) 